\title{
Investigación-acción como recurso para la innovación y mejora de la práctica educativa en conservatorios: una experiencia en las enseñanzas elementales de música en España
}

\author{
Emilia Campayo-Muñoz y Alberto Cabedo-Mas
}

Universitat Jaume I de Castellón

(Recibido, 27 septiembre, 2017; Aceptado, 16 de enero, 2018)

RESUMEN: A menudo, las enseñanzas musicales en los conservatorios de música se basan en metodologías arraigadas a la tradición. Aunque en el currículo de dichas enseñanzas existan asignaturas que tienen como finalidad desarrollar en el alumnado conocimientos y habilidades pedagógicas, estas materias representan una carga lectiva poco significativa en el conjunto del currículo. Como consecuencia, el docente formado en conservatorios no siempre desarrolla en profundidad los conocimientos y habilidades psicopedagógicas adecuadas para la práctica educativa. Con el fin de dar respuesta a esta realidad, el presente artículo tiene como objetivo introducir al profesorado de conservatorio en la metodología de investigación-acción como recurso para la innovación y mejora de la propia práctica educativa. Para cumplir con este objetivo, en primer lugar se realiza una aproximación al concepto de investigación-acción para después presentar un ejemplo de investigaciónacción propia llevada a cabo en el aula de música en conservatorios. Dicha propuesta explora la relación entre las competencias emocionales, las estrategias de aprendizaje autorregulado, la competencia percibida y su influencia en la interpretación musical. Finalmente, se discuten las implicaciones para la formación permanente del profesorado y se formulan una serie de propuestas para ser aplicadas en este contexto de formación.

Palabras clave: Educación musical, investigación-acción, formación del profesorado, competencias emocionales, aprendizaje autorregulado.

\section{Action research as a resource for innovation and educational improvement in music conservatories: an experience in elementary music teaching in Spain}

\begin{abstract}
Teaching in music conservatories is often based on methodologies rooted in traditional teaching methods. Although the music education curriculum sometimes includes modules aimed at developing knowledge and pedagogical skills in the students, these seldom have a significant presence within the curriculum. As a result, the teacher trained in conservatories does not often deeply develop the knowledge and psychopedagogical skills required for educational practice. To address this issue, this paper aims to introduce conservatoire teachers to action research as a resource for improving their own educational practice. In fulfilling this objective, first, the authors define the concept and highlight the importance of action research, and second, introduce an example of action research project carried out in the music classroom in conservatories. This example explores the relationship between
\end{abstract}


emotional skills, self-regulated learning strategies, perceived competence and its influence on musical performance. Finally, the implications for the continuous training of teachers are discussed and a set of proposals are formulated to be applied in this training context.

Keywords: Music education, action research, teacher training, emotional skills, self-regulated learning.

Correspondencia: Alberto Cabedo-Mas. Facultad de Ciencias Humanas y Sociales, Universitat Jaume I. Av. Vicent Sos Baynat, s/n; 12071, Castellón de la Plana. Tel: 964729718. Email: cabedoa@uji.es.

\section{Introducción}

El docente ha de estar en continua formación para adaptarse a los cambios continuos de la sociedad reflejados en el aula (Lucato, 2001). En este sentido, la investigación emerge como una aliada del profesor al favorecer la evolución de su profesión $\mathrm{y}$, consecuentemente, su calidad educativa (Latorre, 2003). La investigación en educación musical es relativamente reciente en comparación con otras áreas de conocimiento. Es por ello que se precisa de una mayor presencia de la investigación en este campo con tal de facilitar la organización y la creación de recursos necesarios para una enseñanza y aprendizaje musical de calidad (Díaz \& Giráldez, 2015). Dentro del ámbito de la educación musical, la investigación se hace especialmente necesaria en los conservatorios de música, debido a que los profesores que imparten enseñanza en esta institución no siempre han recibido una educación pedagógica sólida (Bautista \& Pérez Echeverría, 2008). El currículo de los planes de estudio que regía la formación de los docentes que imparten enseñanza en conservatorios en la actualidad -Plan del Estudios del 66 (Decreto 2518/1966) ${ }^{1}$ y LOGSE (Orden del 28 de agosto de 1992) ${ }^{2}$ - carecía de contenidos psicopedagógicos que permitieran al futuro profesor de música desarrollar las habilidades necesarias para impartir una enseñanza musical de calidad (Pozo, Bautista, \& Torrado, 2008). Sin embargo, a lo largo del siglo XXI, ha surgido una tendencia novedosa y evolutiva que cuestiona y propone nuevos caminos a las metodologías tradicionales utilizadas en los conservatorios de música. Muestra de ello es el monográfico dedicado a las prácticas y concepciones de la educación musical en conservatorios publicado en 2008 la revista Cultura y Educación (ver, por ejemplo, Bautista \& Pérez-Echeverría, 2008; Casas \& Pozo, 2008; Pozo et al., 2008).

Este artículo surge de la necesidad de fomentar la integración de la investigación dentro del ámbito de la educación musical en conservatorios. El objetivo principal es introducir a los docentes que desarrollan su labor docente en esta institución en la metodología de la investigación-acción. Este objetivo se articula a través de la presentación de nuestra propia experiencia con esta metodología, con la que se pretende ofrecer recursos que permitan a la comunidad de profesores de conservatorios mejorar y actualizar su propia práctica educativa. Además, también se abordan cuestiones referentes a la importancia de la formación permanente del profesorado, para que estos profesionales puedan desarrollar los conocimientos y habilidades necesarias para integrar la investigación como parte inherente de su labor docente.

\section{Investigación-acción en el aula de música}

Kemmis y McTaggart (1988) definen esta metodología cualitativa como "una forma de indagación introspectiva colectiva emprendida por participantes en situaciones sociales con 
objeto de mejorar la racionalidad y la justicia de sus prácticas sociales o educativas, así como su comprensión de esas prácticas y de las situaciones en que éstas tienen lugar" (p. 9). La principal finalidad de la investigación-acción es encontrar caminos que permitan, tanto al individuo en particular como a la humanidad en general, vivir el día a día de una forma más sostenible (Kemmis, 2010).

En al ámbito de la educación, la investigación-acción pretende mejorar las propias prácticas educativas, la compresión de las mismas y su contexto (Carr, 1993; Carr \& Kemmis, 1986). Además, representa el compromiso del profesor hacia su propia práctica docente, la cual le servirá de herramienta para realizar cambios de mejora tanto en su propia labor docente como en el currículum (Elliott, 1993).

La investigación-acción es participativa y colaborativa (Kemmis \& MacTaggart, 1988). Esta característica le permite a los profesores aprender los unos de los otros a través de las discusiones planteadas para encontrar soluciones a problemas comunes (Cain, 2011). A su vez, esta metodología ofrece al docente la posibilidad de desempeñar paralelamente los roles de profesor e investigador. Esta característica permite desarrollar una mayor comprensión y conocimiento de su propia práctica docente, lo que favorece la mejora de la misma (Latorre, 2003). Además, siguiendo con este autor, la práctica de la investigación-acción en el aula fomenta: 1) el autodesarrollo personal, 2) una mejor práctica profesional, 3) mejoras en la institución educativa y 4) unas mejores condiciones sociales.

Aunque los descubrimientos provenientes de procesos de investigación-acción no pueden ser generalizables, permiten realizar un estudio comparativo de distintos casos que permiten detectar problemas comunes para ser compartidos entre los diferentes docentes para darles solución (Cain, 2011; Elliott, 1990). Desde esta perspectiva, se puede catalogar la investigación-acción como una modalidad de estudio de casos (Elliott, 1990; Latorre, 2003; McKernan, 1999).

La investigación-acción es una metodología consolidada dentro del campo de la educación (Elliott, 1990). En España, encontramos estudios realizados en diferentes etapas educativas de educación general (Diego-Rasilla, 2007; Domingo \& Marqués, 2013). Algunos de ellos se centran en disciplinas concretas como matemáticas (Romera, 2012), educación física (Fraile, 1995) o música (Casals, Vilar, \& Ayats, 2008), entre otras. Sin embargo, no encontramos estudios basados en procesos de investigación-acción en el ámbito de conservatorios de música. Cain (2008) realizó una revisión de la literatura que incorporaba algunos ejemplos de investigación-acción en contextos de conservatorios fuera de nuestras fronteras (ver, por ejemplo, Bannan, 2004; Gaunt, 2007; Odena \& Cabrera, 2006). Se trata de estudios pioneros de investigación-acción en el ámbito de la educación en conservatorios. Es importante tener presente la herencia tradicionalista que caracteriza a las enseñanzas musicales desarrolladas en estas instituciones (Pozo et al., 2008) para poder entender algunas de las peculiaridades que Cain (2008) resalta de los citados estudios: (1) estaban destinados a mejorar aspectos relacionados con la mejora de la práctica instrumental y (2) estaban planteados para ser desarrollados en un número reducido de sesiones, por lo que, en este sentido, no siguieron la característica cíclica de la investigación-acción. El hecho de que el profesor de conservatorio tenga la iniciativa de emplear la investigación-acción como método de investigación de su propia práctica educativa denota un cambio de paradigma en este tipo de enseñanza. 
Existen diferentes modelos de investigación-acción. Aunque cada uno de ellos presenta sus propias peculiaridades y matices (Bausela, 2004), todos parten del modelo de investigación-acción planteado por el psicólogo Kurt Lewin en 1946. Dicho modelo consiste en una espiral conformada por diferentes ciclos que conlleva investigar y actuar siguiendo unas etapas determinadas: (1) elaborar un plan, (2) ponerlo en marcha y (3) evaluarlo (Latorre, 2003). Kemmis (1989), diseñó un modelo de investigación-acción para aplicarlo a la enseñanza. Este modelo consta de cuatro fases claramente definidas para cada ciclo de acción (Figura 1). Como consecuencia, el modelo de Kemmis (1989) permite al docente-investigador explorar la propia práctica educativa mediante la elaboración e implementación de un plan de acción fácilmente adaptable a la dinámica de las aulas. Por su practicidad y su excelente adaptación a la práctica educativa, el modelo elegido para este estudio es el propuesto por Kemmis (1989).

Figura 1. Fases de la investigación-acción (Kemmis, 1989)

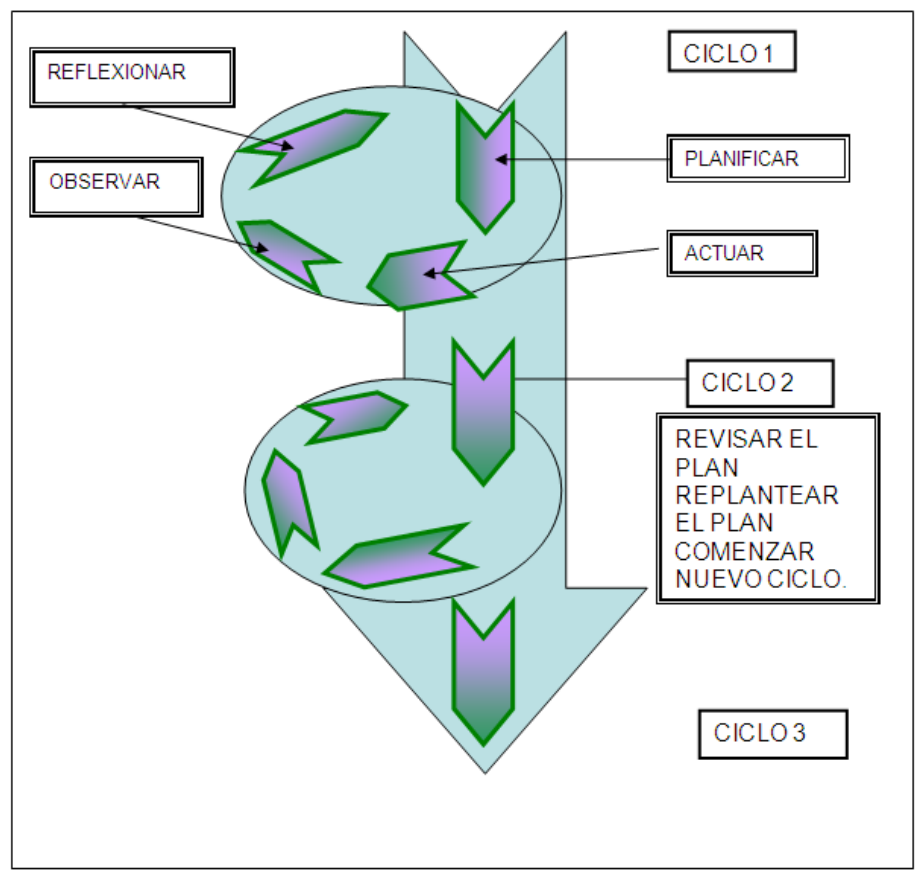

Un ejemplo de proceso investigación-acción en conservatorios: El estudio de la competencia percibida y su relación con la interpretación musical

A continuación presentamos un ejemplo propio de investigación-acción llevado a cabo en las enseñanzas elementales de música en conservatorios. El estudio se centró en explorar en qué medida la competencia percibida influye en la interpretación musical.

\section{Diseñando los orígenes}

Desde el principio de su aprendizaje, el niño se va conformando creencias acerca de sus propias capacidades. Dichas creencias predecirán futuros logros en el contexto del aprendizaje (Austin, Renwick, \& McPherson, 2006). En relación a las creencias, Bandura (1986), define el 
concepto de autoeficacia como "el juicio de las personas acerca de sus capacidades para organizar y ejecutar los cursos de acción requeridos para alcanzar tipos designados de actuaciones" (p. 391). Este concepto tiene estrecha relación con el de competencia percibida, puesto que es uno de los factores que Bermúdez, Rueda y Pérez (2002) identifican como influyente en el constructo de competencia percibida.

Según la Teoría de la Autodeterminación (TAD) (Deci \& Ryan, 1985), la competencia es una de las tres necesidades psicológicas básicas que el individuo necesita experimentar para su bienestar. Todas ellas tienen un componente motivacional (Deci \& Ryan, 2000). La percepción que el niño tiene sobre su propia competencia es importante dentro del ámbito académico, dada la relación existente entre esta y el rendimiento académico (Black \& Wolf, 1990). Los niños que se perciben a sí mismos como competentes muestran mayor motivación por el aprendizaje, utilizan las estrategias y destrezas que conocen, persisten y enfrentan dificultades. Como consecuencia, suelen conseguir las metas que se proponen (Austin et al., 2006). Según la teoría de la atribución causal de motivación y emoción de Weiner (1985, 1987), las creencias que los niños se generan sobre sus propias capacidades están en función de las atribuciones causales que estos realizan sobre sus acciones.

Siguiendo a Bermúdez et al. (2002), la estabilidad emocional es uno de los factores que intervienen en la formación de las propias creencias. Por esta razón, las competencias emocionales, entendidas como la capacidad de poner en práctica la habilidad de ser consciente de nuestras emociones y gestionarlas adecuadamente en su cotidianidad (Bisquerra \& Pérez, 2007), emergen como una herramienta clave para la mejora de la competencia percibida. Algunas competencias emocionales como la autoestima, el enfoque positivo o la autoconfianza están relacionadas con una mejora de la competencia percibida (Dweck, 2000). Las personas que integran el ámbito afectivo de los niños, padres y profesores principalmente son fundamentales para favorecer el desarrollo de dichas competencias emocionales en el alumnado (Campayo \& Cabedo, 2016a; Hallam, 2002). Los niños que están expuestos a críticas negativas de profesores y padres respecto de su rendimiento adoptan emociones negativas, tienen baja evaluación de sus propias acciones, no encuentran soluciones constructivas al problema y tienden a no persistir en la tarea (Dweck, 2000). Además, el ambiente de clase genera un gran impacto en las creencias de los jóvenes adolescentes. Un ambiente favorecedor debería promover seguridad, aceptación y respeto entre iguales así como desafío intelectual (Wigfield \& Eccles, 1994).

La educación musical requiere una gran exigencia, tanto cognitiva como emocional. Por esta razón, cuando nos centramos en la educación musical en conservatorios, ponemos de manifiesto que este tipo de educación debería considerar tanto el desarrollo de las habilidades técnicas e interpretativas propias de la práctica instrumental como aquellas que favorezcan el desarrollo de creencias positivas acerca de dichas habilidades (Pajares, 2002). Además, es conveniente dotar al niño de estrategias que le permitan lograr sus metas, como por ejemplo la concentración, organización, consciencia del hacer o gestión del tiempo (McPherson \& Davidson, 2006). Un alumno cuya percepción de competencia es positiva desarrolla estrategias de autorregulación en el estudio, como son la perseverancia, la iniciativa, la planificación o el afrontar las dificultades adecuadamente (Zimmerman \& Pons, 1988). Esta última estrategia es importante para el éxito en los estudios musicales ya que, según O’Neill y Sloboda (1997), los niños que no se adaptan bien a las dificultades acaban desmotivándose y generando una 
creencia negativa acerca de sus propias capacidades, lo que puede traducirse en un abandono del instrumento.

Sin embargo, las enseñanzas musicales en conservatorios, están fundamentalmente enfocadas al desarrollo de los aspectos técnicos en detrimento del emocionales o psicológicos (Pozo et al., 2008). Es más, la mayoría de profesores de conservatorio no han recibido una formación psicopedagógica adecuada para ayudar al alumnado a enfrentar ciertos estados emocionales propios de las enseñanzas instrumentales como son, por ejemplo, la frustración o la ansiedad escénica (Fernández \& Escoda, 2015). Se trata de un sistema educativo en el que el talento suele ser la herramienta de medición para clasificar y juzgar al alumnado. De esta aptitud va a depender la selección y permanencia en este tipo de enseñanza. Como consecuencia, los aspectos relacionados con la capacidad musical del alumno, como pueden ser el oído, la entonación, la expresión, la técnica instrumental o el sentido del ritmo, incide directamente en su autoconcepto y autoestima (Fernández \& Escoda, 2015). Toda esta serie de normas y expectativas acerca de la idea de lo que debe ser un buen músico en las que se fundamentan las enseñanzas en conservatorios (Pozo et al., 2008) puede desembocar en un estado de ansiedad y angustia a la hora de enfrentar la actuación en público, por lo que toda posibilidad de disfrute queda anulada (Fernández \& Escoda, 2015). Es más, muchos intérpretes tienen la necesidad de ingerir betabloqueantes para controlar los síntomas que la ansiedad de interpretar en público les produce (Bourgeois, 1991; North \& Hargreaves, 2008).

Según lo expuesto hasta el momento, la competencia percibida emerge como una habilidad que influye de forma directa en el rendimiento de una persona con respecto a una tarea en particular. Dadas las implicaciones que esta premisa podría tener en el ámbito académico, decidimos explorar en qué medida el trabajo de la competencia percibida influye en la mejora de la interpretación musical del alumnado de conservatorios.

\section{Aplicando la investigación-acción a nuestro estudio}

Elegimos la metodología de investigación-acción porque nos permitía integrar, de forma paralela, la investigación a nuestra labor docente. Además, nos ofrecía la posibilidad de evaluar nuestra práctica como profesionales de la enseñanza y plantear nuevas fórmulas para mejorarla.

El proceso de investigación-acción lo llevamos a cabo en base a las directrices que Kemmis y McTaggart (1988) proponen. Las fases de la investigación-acción fueron las siguientes:

\section{Planificación: plan de acción}

El plan de acción fue diseñado en base a un objetivo principal: mejorar la competencia percibida del alumnado que cursaba tercer curso de piano de enseñanzas elementales. También se tuvieron en cuenta las características del alumnado. El plan de acción consistió en el diseño ad hoc de una serie de actividades orientadas al trabajo de las estrategias de aprendizaje autorregulado y competencias emocionales indicadas en al Tabla 1 con la finalidad de mejorar la competencia percibida. Junto a las actividades, se especificó la información necesaria para el desarrollo de las mismas:

1. Lugar de implementación. Las actividades podían implementarse en la clase individual de instrumento y/o en la de conjunto. 
2. Momento de aplicación. Este podía ser puntualmente o bien prolongado a lo largo del curso dependiendo de la naturaleza de las actividades. Las actividades transversales fueron desarrolladas de forma paralela al estudio de las obras de repertorio a lo largo de las distintas sesiones, mientras que las específicas se aplicaron de forma puntual para trabajar contenidos concretos relacionados con de la educación emocional o el desarrollo de estrategias de aprendizaje.

3. Técnicas de observación empleadas.

4. Recursos materiales, espaciales y/o humanos.

5. Reflexión acerca de la finalidad de la actividad y expectativas que se pretendían conseguir con la misma.

Tabla 1. Estrategias de aprendizaje y competencias emocionales trabajadas

\begin{tabular}{l|l}
\multicolumn{1}{c|}{$\begin{array}{c}\text { ESTRATEGIAS APRENDIZAJE } \\
\text { AUTORREGULADO }\end{array}$} & \multicolumn{1}{c}{ COMPETENCIAS EMOCIONALES } \\
\hline - Concentración & Competencias intrapersonales \\
- Constancia & - Autoconciencia emocional \\
- Organización & - Nombrar emociones \\
- Disfrute & - Expresión emocional \\
- Gestión de retos & - Control emocional \\
& - Autoestima \\
& - Automotivación \\
& - Autoconfianza \\
& - Responsabilidad \\
& - Enfoque positivo \\
& Competencias interpersonales \\
& - Escucha activa \\
& - Cooperación \\
& - Respeto \\
& - Comunicación expresiva y receptiva \\
& - Empatía \\
\hline
\end{tabular}

La Tabla 2 muestra algunas de la actividades diseñadas en base a las competencias emocionales que consideramos necesarias para desarrollar las estrategias de aprendizaje autorregulado. Cabe señalar que el propósito de este estudio no es validar las actividades planteadas, sino utilizarlas como herramienta para llevar a cabo la acción para cumplir con el objetivo propuesto en la fase de planificación. 
Tabla 2. Algunas actividades diseñadas para el plan de acción

\begin{tabular}{|c|c|c|}
\hline $\begin{array}{c}\text { ESTRATEGIAS } \\
\text { APRENDIZAJE } \\
\text { AUTORREGULADO }\end{array}$ & $\begin{array}{l}\text { COMPETENCIAS } \\
\text { EMOCIONALES }\end{array}$ & ACTIVIDADES \\
\hline \multirow[b]{2}{*}{ Concentración } & $\begin{array}{l}\text { Concienciación } \\
\text { emocional }\end{array}$ & $\begin{array}{l}\text { 1. Ayudar al alumno a ser consciente de cuándo no } \\
\text { estaba concentrado }\end{array}$ \\
\hline & Control emocional & $\begin{array}{l}\text { 2. Realizar respiraciones conscientes antes de } \\
\text { estudiar } \\
\text { 3. Hacer pausas cada determinado tiempo y con } \\
\text { diferentes frecuencias establecidas criterialmente. }\end{array}$ \\
\hline Constancia & Control emocional & $\begin{array}{l}\text { 4. Confeccionar y seguir un horario de estudio } \\
\text { cumplimentado por los propios alumnos y su } \\
\text { respectivos padres a principio de curso. }\end{array}$ \\
\hline \multirow[b]{2}{*}{ Organización } & Control emocional & $\begin{array}{l}\text { 5. Escribir en clase lo que han de hacer y cómo } \\
\text { estudiarlo paso a paso en una libreta }\end{array}$ \\
\hline & $\begin{array}{l}\text { Autoestima, } \\
\text { autoconfianza y } \\
\text { responsabilidad }\end{array}$ & $\begin{array}{l}\text { 6. Estudiar con ellos en clase para que aprendan los } \\
\text { diferentes pasos a seguir al estudiar una obra o } \\
\text { resolver las distintas dificultades que ésta plantea } \\
\text { (identificar el error, proponer diferentes soluciones } \\
\text { para el mismo, etc.) }\end{array}$ \\
\hline Gestión de retos & $\begin{array}{l}\text { Autoestima y } \\
\text { autoconfianza }\end{array}$ & $\begin{array}{l}\text { 7. Hacer consciente al alumno de sus puntos fuertes } \\
\text { y débiles con respecto al problema planteado y } \\
\text { ayudarle a utilizarlos en su beneficio. }\end{array}$ \\
\hline Disfrute & $\begin{array}{l}\text { Concienciación } \\
\text { emocional }\end{array}$ & $\begin{array}{l}\text { 8. Inventar una historia en relación a la emoción que } \\
\text { la obra a interpretar le suscita } \\
\text { 9. Relacionar la obra con alguna experiencia y su } \\
\text { respectiva emoción experimentada por el alumno. }\end{array}$ \\
\hline
\end{tabular}

Además de las actividades diseñadas e implementadas para el plan de acción, se procuró dinamizar las clases realizando actividades variadas. La finalidad de esta estrategia metodológica residía en mantener al alumnado motivado para favorecer una predisposición positiva hacia el aprendizaje. Además, con tal de involucrar a los alumnos en el proceso de enseñanza-aprendizaje y desarrollar su responsabilidad, fueron informados de la utilidad que las estrategias de estudio tenían para mejorar su interpretación musical, así como del papel tan importante que ellos desempañaban para poderlas desarrollar.

\section{$\underline{\text { Acción }}$}

El estudio se articuló a lo largo de un curso escolar. La implementación se desarrolló en las asignaturas de (1) instrumento -la cual se imparte una vez a la semana y es de carácter individual- y (2) conjunto, asignatura en la que los alumnos de la misma especialidad y curso tienen la oportunidad de interpretar música en grupo -también de una hora semanal y de (C) Psy, Soc, \& Educ, 2018, Vol. 10(1) 
carácter grupal. La implementación se realizó de forma paralela y coherente a los contenidos propios del currículo de la asignatura de piano.

El plan de acción fue evaluado al principio de la implementación por una investigadora experta externa. Posteriormente, se realizaron diferentes evaluaciones del mismo por un colega crítico diferente en cada uno de los trimestres. En todos los casos, la evaluación se llevó a cabo mediante observación externa no participante. Las evaluaciones se tuvieron en cuenta para la mejora del plan de acción. Por su parte, la profesora-investigadora también realizó distintas evaluaciones de éste al final de cada uno de los ciclos de acción con el fin de conseguir de forma efectiva el objetivo propuesto.

\section{Observación}

Coincidiendo con Latorre (2003), esta técnica nos permitió estar en contacto directo con el fenómeno estudiado, lo que nos facilitó conocerlo y analizarlo en profundidad. Al igual que en el resto de fases, y siguiendo las recomendaciones de Kemmis y McTaggart (1988), se procuró que la observación también fuera lo suficientemente flexible como para poder recoger de manera organizada tanto los datos previstos como aquellos que surgieron de forma inesperada; todo ello con la finalidad de enriquecer y ampliar la perspectiva de partida (Anguera \& Escolano-Pérez, 2014). Esta fase constituyó la base documental para la reflexión posterior, por lo que el control de la acción y la recogida de datos se llevaron a cabo de una forma sistemática y rigurosa.

\section{$\underline{\text { Reflexión }}$}

La reflexión nos permitió detectar, a lo largo del proceso de investigación-acción, los aspectos del plan de acción que no resultaron ser efectivos para la consecución del objetivo propuesto. Además, ofreció la posibilidad de identificar nuevos problemas que surgieron durante la implementación con los que no contábamos en un primer momento. Posteriormente, y con base en la reflexión, se realizaron los cambios pertinentes que conformaron un plan de acción renovado y listo para ser aplicado en el siguiente ciclo de acción. En la reflexión también se consideraron no solo nuestra perspectiva, sino la aportada por la experta y los colegas críticos que participaron en la investigación a los que nos referimos en el anterior apartado. Algunas de las observaciones de estos participantes que sirvieron de utilidad para conferir al plan mayor efectividad fueron:

1. Participación activa del alumno a la hora de apuntar lo que tenía que hacer pues, al principio de la implementación del plan de acción, era la profesora quien realizaba esta tarea.

2. Realizar ejercicios fuera del instrumento con tal de resolver problemas técnicos.

3. Cambiar de actividad cuando se detecte que el alumno está cansado o desconcentrado.

4. Aumentar el tiempo para que el alumno reflexione sobre las cuestiones relativas a la resolución de pasajes.

5. Poner ejemplos de todo lo que se explica.

6. Explicar la finalidad de cada una de las actividades realizadas.

Algunos de los puntos positivos del plan de acción que fueron detectados por la mayoría de los observadores a lo largo de todo el proceso, y que fueron consolidados en el mismo fueron:

1. Reforzar las actitudes positivas.

2. Emplear vocabulario en positivo. 
3. Motorizar al estudiante para que encuentre las soluciones a los problemas por sí mismo.

4. Empleo de actividades variadas.

\section{Seleccionando participantes}

La selección de los alumnos participantes en esta investigación quedó limitada a aquellos que estaban matriculados en la asignatura de piano con la profesora-investigadora y que coincidían en el resto de asignaturas grupales (conjunto, lenguaje musical y coro). Los estudiantes que cumplían estos requisitos fueron tres (Juan, María y Laura), por lo que se realizó un estudio de casos desarrollado como un proceso de investigación-acción. Todos ellos estudiaban tercer curso de piano de enseñanzas elementales de música y tenían 11 años de edad al comenzar la investigación. Sin embargo, uno de los participantes, Laura, cumplía 12 años al finalizar el año, por lo que era mayor e iba un año más adelantada que el resto en la educación obligatoria. Los estudiantes provenían de familias acomodadas económicamente y con diferentes niveles de formación académica. Además del alumnado que participó de forma activa, también estuvieron involucrados sus respectivos padres, el profesor de lenguaje musical, la profesora de coro y algunos compañeros con los que compartían las asignaturas grupales. La profesora de las asignaturas de piano y conjunto, fue quien llevó a cabo la investigación.

\section{Escogiendo instrumentos de recogida de datos}

Los instrumentos de recogida de datos utilizados fueron los siguientes:

1. Entrevista. Se realizó un ciclo de entrevistas semiestructuradas antes y después del estudio a los alumnos participantes, a sus padres y al profesor de lenguaje musical (asignatura grupal en la que coincidían los tres alumnos participantes en el estudio). La finalidad de las entrevistas era conocer el punto de partida y la evolución acontecida durante el desarrollo de la investigación con respecto a las estrategias de aprendizaje y las competencias emocionales de los estudiantes implicados en el estudio.

2. Diario del profesor. Documento en el que la profesora de piano y conjunto reflejaba por escrito los hechos más significativos acontecidos durante la clase (Porlán \& Martín, 1991) en relación a la competencia percibida de los estudiantes y su influencia en el rendimiento académico.

3. Videos. Se realizó una grabación mensual a lo largo de todo el curso tanto de la asignatura de piano como de la de conjunto. Los vídeos nos permitieron observar y comprender con mayor detalle algunos aspectos relacionados con la competencia percibida de los estudiantes y su influencia en la mejora en la interpretación.

\section{Analizando la información recogida durante la implementación del plan de acción}

Los datos fueron analizados cualitativamente. Las entrevistas transcritas, el diario del profesor y las grabaciones de las distintas sesiones fueron registradas en el programa informático MAXQDA 12 para su análisis de contenido. El proceso de categorización fue inicialmente realizado de manera independiente por los dos investigadores involucrados en el estudio. La categorización se llevó a cabo deductivamente: se partió de la categoría principal "competencia" basada en una de las tres competencias básicas definidas por (Deci \& Ryan, 1985) en su Teoría de la Autodeterminación (TAD). Dicha categoría se desglosó en una serie 
de subcategorías (concentración, constancia, organización, disfrute y gestión de retos), que consistían en las estrategias desarrolladas durante el plan de acción con la finalidad de mejorar la competencia percibida del alumnado respecto a su propia práctica del instrumento. Este proceso de categorización se realizó en base al sistema categorial del que dispone MAXQDA 12. De esta forma, se organizó la información en función de su naturaleza temática. El procedimiento consistió en la categorización de los distintos datos (textos y vídeos) para, posteriormente, extraer los sumarios de las distintas categorías establecidas mediante la herramienta de elaborar matrices por cada categoría seleccionada. Esto permitió cruzar la información a través del proceso de triangulación hermenéutica (Cisterna, 2005).

La investigación tuvo en cuenta las normas éticas de confidencialidad, autorización por parte de padres y de centro educativo y conservación segura de datos de las personas participantes.

\section{Explorando la información obtenida}

A continuación, presentamos un análisis de las estrategias de aprendizaje trabajadas en base a la evolución de cada uno de los casos estudiados. Cabe señalar que los resultados presentados corresponden a las tendencias observadas en tres alumnos particulares y, por esta razón, en ningún caso pretenden ser generalizables. Además, puntualizamos que la finalidad de los resultados es describir lo observado durante el desarrollo del plan de acción sin realizar una valoración acerca de la efectividad y validez de las distintas actividades implementadas como parte de dicho plan.

Concentración

Observamos una relación positiva entre la concentración y la mejora en la efectividad del aprendizaje. Cuando la concentración es alta, el aprendizaje es más efectivo y viceversa:

Diario del profesor (12/11/14): Juan muestra mala memoria y es muy despistado (creo que todo es por la dificultad en la concentración, y no llega a aprenderse las cosas por comprensión, sino por repetición). La consecuencia es que lo estudiado en un día se pierde al siguiente y cuesta avanzar

Diario del profesor (29/04/15): Laura sigue sin rendir como antes. Obviamente, está pasando por una fase en la que le cuesta concentrarse y sacar partido del estudio.

Juan fue quien presentó mayor problema para mantener la concentración durante las clases al principio de la investigación. Sin embargo, es precisamente este alumno quien mejoró su concentración significativamente a lo largo del curso:

Profesora: ¿Y ha mejorado su concentración en clase, o crees que sigue igual que al principio?

Profesor de lenguaje musical: No, no, sí [con seguridad]. Está más a la faena.

Esta mejora repercutió favorablemente en su competencia percibida y rendimiento al estudiar: 
Diario del profesor (10/06/15): Me he dado cuenta que el hecho de desarrollar su capacidad de concentración y organización ha ido aumentando su propia autoestima, lo cual lo ha llevado a rendir más en lo que hace, pues se siente más capaz de hacer las cosas.

Aunque la evolución de cada uno de los casos fue distinta, un punto en común fue la dificultad de mantener la concentración durante el estudio durante períodos de tiempo prolongados:

Padre María: Aprovecha muy bien el tiempo, pero sí que es verdad que si tiene un tiempo marcado muy largo, se levanta muchas veces.

Mientras que la concentración de María se mantuvo estable durante todo el curso, la de Laura experimentó un descenso, lo que repercutió en su efectividad al tocar el instrumento. No se pueden obviar las circunstancias de Laura como una posible explicación a la creciente desconcentración que sufrió a lo largo del plan de acción: por un lado, los cambios físicos propios de la entrada en la adolescencia (es la alumna de mayor edad de los tres casos estudiados), y por otro, los académicos al cambiar de primaria a secundaria.

\section{Constancia}

En general, apreciamos una relación positiva entre el desarrollo de la constancia y una mejora en los resultados de las distintas asignaturas:

Diario del profesor (18/03/2015): Con Juan estoy muy contenta. Los demás profesores también me han dicho que ha cambiado mucho de actitud, y que ya trae las cosas estudiadas a clase, y ha mejorado mucho. La verdad es que está mucho más motivado que a principio de curso. Yo creo que es porque se ve más capaz de hacer todo lo relacionado con la música, con las enseñanzas musicales, cosa de la que no estaba demasiado confiado al principio.

En este caso, se puede observar cómo la confianza en las propias posibilidades son importantes para que la motivación aumente $\mathrm{y}$, a su vez, reforzar la autorregulación del aprendizaje.

Al igual que ocurrió con la concentración, María mantuvo estable su constancia en el estudio. Sin embargo, la constancia de Laura fue disminuyendo progresivamente a lo largo del curso. Muestra de ello son las continuas referencias reflejadas por la profesora en su diario:

Diario del profesor (29/10/2014): Laura no había estudiado mucho esta semana Diario del profesor (10/12/2014): Lleva como un mes un poco descolocada

Diario del profesor (20/05/2015): Laura sigue en su línea de últimamente: no estudia como debe pese a que sabe lo que tiene que hacer.

Llama la atención que los dos alumnos que siguieron el horario de estudio confeccionado a principio de curso mejoraron su constancia durante el plan de acción: 
Profesora: ¿Has seguido el horario de curso que hicimos a principio de curso?

Laura: No mucho.

Profesora: [ríe]. ¿Tú crees que si lo hubieras seguido te hubiera ayudado a estudiar de una forma más constante?

Laura: Sí.

Profesora: ¿Y por qué lo crees?

Laura: Porque así no me hubiera pasado lo que me ha pasado.

Profesora: ¿Has seguido el horario de estudio que hicimos a principio de curso?

María: Sí.

Profesora: ¿Y crees que haberlo seguido te ha ayudado a ser más constante?

María: Sí.

Profesora: ¿Y has seguido el horario de curso que hicimos a principio de curso?

Juan: Sí.

Profesora: ¿Y crees que haberlo seguido te ha ayudado a ser más constante en el estudio?

Juan: No sé.

Sin embargo, cabe indicar que una de las razones por la que Laura no siguió el horario de estudio fue debido a que sus padres no pudieron atender los momentos de estudio de Laura, por motivos de trabajo:

Madre Laura: Y si un día llegas a las 21:30 ó las 22:00 h, parece que los vecinos...También al medio día, si vives en un bloque de pisos sabes que hay personas que trabajan a turno... y parece que se sepa mal a esas horas que se ponga. En ese sentido ha sido un poco culpa nuestra, la verdad.

Padre Laura: Sí, sí. Y además cuando coincidimos los dos trabajando, pues entonces se hacen cargo los familiares, los abuelos, etc. Entonces...

Madre Laura: Es un poco complicado, pero bueno...

\section{Organización}

La organización, junto con la concentración y la organización, emerge como una estrategia de aprendizaje importante para mejorar la efectividad en el estudio. Muestra de ello son los casos de María y Juan, quienes estudiaban de una forma desorganizada al principio del plan de acción. En ambos casos existía una tendencia a querer hacer las cosas rápido y sin fijarse en los detalles lo que, en muchas ocasiones, derivaba en un estancamiento de las obras en el mejor de los casos:

Diario del profesor (24/4/15): si la dejo a su aire, a María le cuesta ver el orden y estudiar de forma organizada, y el resultado es un tanto caótico.

Sin embargo, en los dos casos se fue produciendo una mejora a lo largo del curso: 
Madre María: Igual era más leer lo que era la partitura, ¿no? Y no tan al detalle de las posiciones o de pensar que podía sentir la música... y como su forma de ser es, "bueno, yo hago esto y ya está", y tiene prisa por saber cómo suena cada cosa antes de tenerlo bien aprendido. Es muy impaciente para eso, ¿no? Entonces este año sí que es verdad que lo ha cambiado mucho.

Diario del profesor (19/11/2015): Juan aguanta mejor las clases y viene con buena predisposición. Parece que estudia de una forma más organizada y va obteniendo mejores resultados.

Por su parte, aunque Laura mostró una tendencia a estudiar de forma organizada, su tiempo de estudio no resultó eficaz. Quizás la disminución de su concentración y constancia en el estudio pudo repercutir negativamente en sus resultados.

Gestión de retos

Observamos una relación positiva entre la forma de gestionar los retos y las creencias acerca de las habilidades propias (competencia percibida) con respecto a las enseñanzas musicales. Quizás el caso más significativo sea el de María, quien desde el principio mostró una gran confianza en sus capacidades para afrontar los estudios musicales, lo que favoreció que afrontara las dificultades desde un enfoque positivo:

Padre de María: En la $2^{\mathrm{a}}$ audición del año pasado salió y se atascó a mitad de la pieza... Quiso volver atrás, y se volvió a atascar en el mismo sitio, y al final se levantó, se bloqueó y bajó llorando y ya... Y la siguiente vez era aquello de "¿me volverá a pasar lo mismo...?", pero salió y tocó.

En un principio, Laura mostró dudas acerca de su capacidad con respecto a sus habilidades instrumentales. Sin embargo, estas dudas se fueron desvaneciendo. Una posible explicación de esta mejora es que la profesora le transmitió progresivamente su confiaza en ella y en sus posibilidades con respecto al piano:

[La alumna termina de tocar]

Profesora: ¿Entiendes lo que tienes que hacer?

Laura: Sí. Pero es un poco complicado, ¿no?

Profesora: No, no es complicado. Es solo "pillarle el tranquillo". Luego no tienes ni que pensarlo, ¿vale?

Laura: [Asiente con la cabeza] [Fragmento vídeo 25/03/2015]

Por último, Juan no mostró una actitud positiva ante las dificultades. De hecho, cuando se le presentaba una nueva dificultad se desmoralizaba porque no se veía capaz de realizarlo. Esto retrasó todavía más su aprendizaje:

Profesora: A ver qué podemos hacer con el acorde de "la menor".

Juan: [Comienza a tocar con inseguridad]... No lo sé. 
Profesora: Sí sabes. No te compliques la vida. Que sea sencillo.

Juan: [Lo vuelve a intentar y se para quejándose]

Profesora: Da igual si no te sale el ritmo que tenías pensado. Lo que salga está bien.

Juan: [Vuelve a intentarlo con mayor seguridad y tocando más tiempo]. [Se para...]. No sé [se lleva una mano a la cabeza].

Profesora: ¡Sí sabes! ¡Estaba muy bien! [Fragmento vídeo 26/03/2015]

Este un claro ejemplo de cómo una falta de autoconfianza en un ámbito concreto afecta negativamente en la forma de abordar las dificultades que se presenten en dicho ámbito. Aunque Juan mejoró ligeramente este aspecto, mantuvo esta tendencia durante todo el curso.

Disfrute

Según Laura argumentó en la entrevista, venir al conservatorio era para ella era una vía de escape de la rutina del colegio, donde podía relajarse. Sin embargo, su autoexigencia le impedía en muchas ocasiones disfrutar de lo que hacía:

Diario del profesor (12/02/2015): Hoy han tocado una obra de las que llevan en clase individual. En general, he podido ver cómo ellos se exigen a la hora de tocar (especialmente Laura y otra estudiante de piano), y cómo esto puede ser contraproducente y acrecienta el sufrimiento.

Esta autoexigencia podría tener sus raíces en la forma en la que su padre le transmitió la responsabilidad por los estudios:

Madre Laura: Yo creo que también es porque su padre le ha inculcado desde pequeñita a ser responsable con los estudios. A ser responsable, primero los estudios y luego yo...

Padre Laura: Quizás, a lo mejor, he pecado en ese sentido, y se he sido demasiado exigente.

Una vez detectada la posible causa de la autoexigencia y falta de disfrute de la alumna se le comunicó a los padres, quienes adoptaron las medidas pertinentes para que Laura pudiera equilibrar su nivel de autoexigencia y responsabilidad. Tras este reajuste, se observó una mejora en el disfrute de la estudiante:

Madre Laura: ... la veo...un poquito más suelta.

Padre Laura: La postura que tiene... se nota que siente la música, es decir, cuando un músico siente la música los gestos que hace con el instrumento, pues se ve. Antes estaba más recta. Antes tenía la postura típica de recta. Ahora siente la música, toca el piano y siente... [pausa].

María no presentaba ese nivel de sufrimiento. Sin embargo, tocaba de forma poco natural por estar pendiente de no fallar ninguna nota. Este aspecto lo mejoró poco a poco hasta ir interpretando de forma más natural y suelta. En estos dos casos, el disfrute favoreció una 
actitud más relajada y suelta ante el instrumento, lo que tuvo como consecuencia en una mejora de la interpretación pianística.

Por su parte, Juan comenzó sin ningún interés por los estudios musicales, por lo que éstos no suponían un disfrute para él. Sin embargo, la dimensión social que supuso para él venir a clases en el conservatorio, le despertó el interés por las mismas. Además, al ser un niño muy creativo, las actividades que le ayudaron a desarrollar esta faceta suya también le ayudaron a incrementar su autoestima y sentirse más motivado por la música:

Diario del profesor (18/02/2015): he notado que funciona muy bien cuando la clase es variada, por lo que el hecho de realizar ejercicios antes y después de tocar y hacer algo creativo durante la clase, hace que se vuelva agradable para él y tenga mejor predisposición para aprender.

\section{Formulando conclusiones}

En primer lugar, nos gustaría aclarar que no se pretende presentar generalizaciones a partir de los resultados obtenidos, sino que estos se presentan con la intención de mostrar cómo se sucedieron los hechos durante nuestra experiencia al aplicar la investigación-acción. Partiendo de esta premisa, presentamos las siguientes observaciones:

1. El trabajo de las competencias emocionales favorecen el desarrollo de la competencia percibida.

2. Aunque se aprecia una relación entre las competencias emocionales, las estrategias de aprendizaje autorregulado y la competencia percibida, no siempre se obtiene una mejora en el rendimiento musical.

3. Para que el alumno pueda mejorar su competencia percibida, es necesario establecer un ambiente de confianza en clase y en la relación profesor-alumno.

Dado que las distintas observaciones realizadas en este estudio giran en torno al trabajo de las competencias emocionales, creemos que sería conveniente diseñar y evaluar programas de educación emocional con tal de comprobar su efectividad para su aplicación.

\section{Reflexiones generales}

El principal objetivo de este artículo es introducir al profesor de música de conservatorios en el conocimiento y práctica de la investigación-acción a partir de nuestra experiencia con esta metodología. La característica de la investigación-acción que permite al docente realizar los roles de profesor e investigador al mismo tiempo (Latorre, 2003) ha sido sumamente enriquecedora en nuestro caso. Este tipo de práctica pedagógica nos ha permitido realizar un crecimiento simultáneo y conjunto tanto en el ámbito de la investigación como en el de la docencia. El hecho de realizar un planteamiento riguroso en la observación, recogida y análisis de datos nos permitió detectar qué elementos ayudaron a la mejora de nuestra práctica educativa a lo largo de todo el proceso. La investigación-acción nos ha permitido aprender en profundidad sobre nuestra propia práctica docente: hemos tenido la oportunidad de reflexionar y cuestionar los métodos y estrategias de enseñanza utilizadas de forma habitual. La inherente necesidad existente entre la investigación-acción y el conocimiento del contexto en el que se aplica (Kemmis \& McTaggart, 1988) nos ayudó a desarrollar una práctica docente más 
consciente y humanista. De hecho, la posibilidad de conocer a cada uno de los alumnos en profundidad fue una de las consecuencias más enriquecedoras y gratificantes de nuestra experiencia. Explorar las fortalezas y debilidades de cada alumno, tanto en el ámbito personal como en el de los estudios musicales, sus miedos, sus percepciones de sí mismos, sus ilusiones o sus gustos, dotan de sentido completo a nuestra función como educadores.

Fue gratificante observar la evolución de cada uno de los casos estudiados. Las diferentes características observadas en los procesos de cada alumno pusieron de manifiesto la singularidad de cada individuo y, con ello, la dificultad de realizar afirmaciones generales. Aunque no en todos los casos se apreció una mejora en el rendimiento en el estudio, todos ellos se sintieron protagonistas de su propio proceso de aprendizaje. En todo momento se procuró darles $\mathrm{VOZ}$ con la finalidad de conocer sus necesidades educativas e integrarlas en el plan de acción, el cual fue diseñado para ser lo suficientemente flexible y adaptable a las mismas (Kemmis \& McTaggart, 1988). No podemos dejar de señalar que desempeñar la práctica docente con la intención de desarrollar las competencias emocionales del alumnado, ayudó a crear un ambiente de seguridad para el alumnado (Campayo \& Cabedo, 2016a). Es más, en nuestra experiencia, y coincidiendo con Wigfield y Eccles (1994), el hecho de haber favorecido un ambiente en el que los alumnos se sintieran confiados y respetados por sí mismos y no por sus resultados, les predispuso positivamente hacia los estudios musicales.

Cuando somos conscientes de cómo nuestra intervención como docentes puede influir en las vidas de los estudiantes, ayudándoles a desarrollarse como personas y como profesionales, es cuando la investigación-acción emerge como una necesidad inmediata en las aulas de cualquier nivel educativo. Es por esta razón y por la carencia de formación específica del profesorado de conservatorios para realizar investigación-acción, que este artículo puede resultar interesante y útil a este colectivo de la comunidad educativa. La investigación-acción podría suponer, para la enseñanza musical en conservatorios, la oportunidad de salir del tradicionalismo y evolucionar hacia un tipo de enseñanza más holística y humanista que contribuya al cambio social. Sin embargo, este cambio de paradigma tiene consecuentes implicaciones para la formación del profesorado, tanto a nivel inicial como permanente, así como una reflexión y revisión en profundidad del currículo de las enseñanzas musicales.

\section{Proyecciones en la práctica}

Tal y como queda reflejado en el Real Decreto $1577 / 2006^{3}$, en el que se establece el currículo para las enseñanzas profesionales de música en conservatorios para toda la nación, el objetivo principal de dichas enseñanzas es desarrollar las habilidades técnicas y cognitivas necesarias para la práctica del instrumento. Sin embargo, el trabajo de las habilidades que favorezcan el éxito académico y personal del alumnado no se encuentran concretados en los objetivos y contenidos recogidos en el citado documento (Campayo \& Cabedo, 2013). Si a este aspecto le sumamos la carencia de recursos psicopedagógicos presentes en formación del profesorado de conservatorios (Fernández \& Escoda, 2015), el resultado es un docente que tiene dificultades para practicar una educación holística que atienda a las necesidades del individuo en su conjunto y que le permita desenvolverse con éxito en la vida cotidiana (Campayo \& Cabedo, 2016b). En este sentido, el conocimiento y práctica de la investigaciónacción ofrece al docente de conservatorio la oportunidad de explorar y evaluar su propia 
práctica docente, en aras de contribuir a un cambio de paradigma que contribuya al cambio social. La formación del profesorado es una herramienta valiosa para introducir al profesorado en esta metodología.

En las dos últimas reformas educativas realizadas en España $\left(\mathrm{LOE}^{4}\right.$ y $\left.\mathrm{LOMCE}^{5}\right)$, se subraya la necesidad de desarrollar al máximo el potencial y las habilidades de cada alumno a través del trabajo de las diferentes dimensiones del individuo. Sin embargo, para que esto sea posible, es necesario que el profesorado disponga de los recursos necesarios para favorecer que el alumnado desarrolle las competencias que propugnan dichas reformas educativas (Bautista \& Ortega-Ruíz, 2015). Investigaciones previas señalan que la formación inicial tienen deficiencias significativas en la capacitación de los futuros docentes de música (Pozo et al., 2008). Por ello, para tal fin, son necesarios seguir dos requisitos. Por un lado, una revisión en profundidad del currículum que habilita al futuro docente como profesional de la enseñanza (Bautista, Tan, Ponnusamy, \& Yau, 2016) y, por otro lado, plantear una efectiva formación permanente del profesorado (Borko, 2004). Para que la formación del profesorado sea provechosa, es fundamental que esta (1) aporte ideas prácticas, específicas y concretas a la vez que lo suficientemente flexibles para adaptarse a cualquier situación y contexto (Bautista \& Ortega-Ruíz, 2015; Bautista et al., 2016); (2) se desarrolle en base a las necesidades y preferencias del profesorado y (3) se plantee como un proceso continuo en el que los docentes realicen un trabajo activo y participativo (Bautista \& Ortega-Ruíz, 2015).

La investigación-acción se caracteriza por ser, entre otros aspectos, práctica, participativa, colaborativa y flexible (Latorre, 2003). Estas características favorecen que los docentes desarrollen las competencias adecuadas para dar respuesta y adaptarse a las distintas y variadas demandas sociales con las que éstos se encontrará en el aula. Según (Knight, 2002), este aspecto resulta fundamental para el desarrollo de una buena práctica docente. En base a esta premisa, consideramos que la introducción a la investigación-acción debería formar parte de los programas de formación permanente de todos los niveles educativos, incluyendo en ella a la docencia en conservatorios y escuelas de música.

\section{Propuestas a partir de la propia práctica}

Con la finalidad de introducir al claustro de profesores en la práctica de la investigaciónacción, proponemos la realización de seminarios a nivel de centro a principio de curso. Estos seminarios pueden impartirse bien por algún compañero que tenga experiencia en la práctica de esta metodología, o por personal docente e investigador especializado en investigación-acción procedente de las universidades o de centros de investigación o formación del profesorado. Estos seminarios deben reforzarse a través de cursos de formación del profesorado de carácter eminentemente práctico. Se plantea la estructura de dichos cursos en dos partes distintas y complementarias: formación propiamente dicha y aplicación práctica. La primera fase pretende capacitar al docente en los fundamentos teóricos de la investigación-acción. La segunda fase debe estar destinada a la aplicación práctica de esta metodología en las aulas de música de los docentes participantes. Este planteamiento refuerza los conocimientos adquiridos en la parte teórica al mismo tiempo que se desarrollan las habilidades necesarias para implementar la investigación-acción. Con tal de que el profesorado obtenga un reconocimiento por la actividad realizada, ésta puede desarrollarse dentro del marco de los grupos de trabajo. Una sugerencia 
contempla la participación activa de profesores de instrumento de diferentes especialidades de forma colaborativa y con objetivos comunes. Posteriormente, es importante contrastar los resultados obtenidos y observar los efectos de la investigación-acción en las distintas especialidades instrumentales.

\section{Valorando nuestra experiencia...}

Tras nuestra experiencia con esta metodología, animamos a los profesores de música, y especialmente a los del ámbito de los conservatorios, a que se introduzcan y experimenten con en el campo de la investigación-acción. Se trata de una metodología que fomenta la creatividad, la autonomía, la responsabilidad y una serie de competencias tan valiosas para la educación como para la vida. En nuestro caso, ha sido una experiencia realmente enriquecedora que nos ha ayudado a conocer y transformar nuestra práctica educativa constantemente, al mismo tiempo que hemos aprendido de nosotros mismos.

\section{Agradecimientos}

Este estudio se lleva a cabo en el marco del proyecto de investigación "Educación musical en el currículo obligatorio: análisis y diseño de materiales para una educación en valores", ref. P1·1A2015-01, de la Universitat Jaume I.

\section{Notas}

1 Decreto 2518/1966, sobre reglamento general de los Conservatorios de Música. (BOE de 24 de octubre de 1966).

2 Orden del 28 de Agosto de 1992, por la que se establece el currículum de los grados elemental y medio de música y se regula el acceso a dichos grados. (B.O.E. del 9 de septiembre de 1992).

${ }^{3}$ Por el que se fijan los aspectos básicos del currículo de las enseñanzas profesionales de música reguladas por la Ley Orgánica 2/2006, de 3 de mayo, de Educación. (B.O.E. del 20 de enero de 2007).

${ }^{4}$ Ley Orgánica, 2/2006, de Educación. (BOE de 4 de mayo de 2006).

5 Ley Orgánica 8/2013, para la mejora de la calidad educativa. (BOE de 10 de diciembre de 2013).

\section{Referencias}

Anguera, M., \& Escolano-Pérez, E. (2014). Aplicaciones de la metodología observacional en educación infantil. International Journal of Developmental and Educational Psychology, 4(1), 227-228. doi: 10.17060/ijodaep.2014.n1.v4.606

Austin, J., Renwick, J., \& McPherson, G. E. (2006). Developing motivation. In G. E. Mcpherson (Ed.), The child as musician (pp. 213-238). New York: Oxford University Press.

Bandura, A. (1986). Social Foundations of Thought and Action: A Social cognitive Theory, Englewood Cliffs. New York: Prentice Hall. 
Bannan, N. (2004). A role for action research projects in developing new pedagogical approaches to aural and musicianship education. In J. W. Davidson (Ed.), The music Practitioner: Research for the Music Performer, Teacher and Listener (pp. 295-308). Aldershot: Ashgate.

Bausela, E. (2004). La docencia a través de la investigación-acción. Revista Iberoamericana de Educación. Retrieved from http://s3.amazonaws.com/academia.edu.documents/45393698 /Bausela-4.pdf?AWSAccessKeyId=AKIAJ56TQJRTWSMTNPEA\&Expires=1483557067\& Signature=E14THUSA7gltsRKGexY194dbyG0\%3D\&response-content-disposition=inline \%3Bfilename\%3DLA_DOCENCIA_A_TRAVES_DE_LA_INVESTIGACIO

Bautista, A., \& Pérez Echeverría, M. P. (2008). ¿Qué consideran los profesores de instrumento que deben enseñar en sus clases? Cultura Y Educación, 20(1), 17-34. doi: $10.1174 / 113564008783781477$

Bautista, A., \& Ortega-Ruíz, R. (2015). Teacher professional development: International perspectives and approaches. Psychology, Society \& Education, 7(3), 240-251.

Bautista, A., Tan, L. S., Ponnusamy, L. D., \& Yau, X. (2016). Curriculum integration in arts education: connecting multiple art forms through the idea of "space." Journal of Curriculum Studies, 48(5), 610-629. doi: 10.1080/00220272.2015.1089940

Bermúdez, J., Rueda, B., \& Pérez, A. M. (2002). Estudio de la competencia percibida a partir de sus dos componentes: expectativa de autoeficacia y expectativa de resultados. Revista de Psicología General Y Aplicada: Revista de La Federación Española de Asociaciones de Psicología, 58(1), 75-88.

Bisquerra, R., \& Pérez, N. (2007). Las competencias emocionales. Educación XX1, 10, 61-82. doi: 10.5944/educxx1.1.10.297

Black, N., \& Wolf, A. (1990). Knowledge and competence: current issues in training and education. (H. Black \& A. Wolf, Eds.). London: Careers \& Occupational Information Centre.

Borko, H. (2004). Professional development and teacher learning: Mapping the terrain. Educational Research, 33(8), 3-15.

Bourgeois, J. A. (1991). The management of performance anxiety with beta-adrenergic blocking agents. Jefferson Journal of Psychiatry, 9(2), 13-28.

Cain, T. (2008). The characteristics of action research in music education. British Journal of Music Education, 25(3), 283. doi: 10.1017/S0265051708008115

Cain, T. (2011). How trainee music teachers learn about teaching by talking to each other: an action research study. International Journal of Music Education, 29(2), 149-154. doi: $10.1177 / 0255761410396961$

Campayo, E. A., \& Cabedo, A. (2013). El desarrollo de las competencias intrapersonales a través del aprendizaje de un instrumento musical. Fórum de Recerca, (18), 423-440.

Campayo, E. A., \& Cabedo, A. (2016a). How parents' and teachers' emotional skills foster academic performance in shool music students. Victorian Journal of Music Education, (1), 9-14.

Campayo, E. A., \& Cabedo, A. (2016b). Música y competencias emocionales: posibles implicaciones para la mejora de la educación musical. Revista Electrónica Complutense de Investigación En Educación Musical - RECIEM, 13, 124-139. doi: 10.5209/RECIEM.51864

(C) Psy, Soc, \& Educ, 2018, Vol. 10(1) 
Carr, W. (1993). Calidad de la enseñanza e Investigación-Acción. Sevilla: Díada Editora.

Carr, W., \& Kemmis, S. (1986). Becoming critical: Education, knowledge and action research. Londres: Falmer.

Casals, A., Vilar, M., \& Ayats, J. (2008). La investigación-acción colaborativa: Reflexiones metodológicas a partir de su aplicación en un proyecto de Música y Lengua. Revista Electrónica Complutense de Investigación En Educación Musical, 5(4), 1-17.

Casas, A., \& Pozo, J. I. (2008). ¿Cómo se utilizan las partituras en la enseñanza y el aprendizaje de la música? Cultura y Educación, 20(1), 49-62. doi: $10.1174 / 113564008783781503$

Cisterna, F. (2005). Categorización y Triangulación como Procesos de Validación del Conocimiento en Investigación Cualitativa. Theoria, 14(1), 61-71.

Deci, E. L., \& Ryan, R. M. (1985). The general causality orientations scale: Self-determination in personality. Journal of Research in Personality, 19, 109-134. doi: 10.1016/00926566(85)90023-6

Deci, E. L., \& Ryan, R. M. (2000). The "What" and "Why" of Goal Pursuits: Human Needs and the Self-Determination of Behavior. Psychological Inquiry, 11(4), 227-268. doi: 10.1207/S15327965PLI1104_01

Díaz, M., \& Giráldez, A. (2015). Music Education in Spain: Beyond the School Curriculum. In S. Figuereido, J. Soares \& R. Schambeck (Eds.), The Preparation of Music Teachers: a Global Perspective. Porto Alegre: ANPPOM - Associação Nacional de Pesquisa e PósGraduação em Música.

Diego-Rasilla, F. J. (2007). La investigación-acción como medio para innovar en las ciencias experimentales. Puls, (30), 103-118.

Domingo, M., \& Marqués, P. (2013). Práctica docente en aulas 2.0 de centros de educación primaria y secundaria de España. Revista de Medios Y Educación, (42), 115-128.

Dweck, C. S. (2000). Self Theories: Their Role in Motivation, Personality, and Development. Lillington: Psychology Press.

Elliott, J. (1990). La investigación-acción en educación. Madrid: Ediciones Morata.

Elliott, J. (1993). El cambio educativo desde la investigación-ación. Madrid: Ediciones Morata. Fernández, B., \& Escoda, F. (2015). La Psicología de la Música y el Miedo Escénico en los Conservatorios: Una Realidad en la Sombra. In II Congreso Nacional de Conservatorios Superiores de Música (pp. 34-49).

Fraile, A. (1995). La investigación-acción: instrumento de formación para el profesorado de educación física. Apunts: Educación Física Y Deportes, (42), 46-52.

Gaunt, H. (2007). Learning and teaching breathing and oboe playing: action research in a conservatoire. British Journal of Music Education, 24(2), 207-231. doi: $10.1017 / \mathrm{S} 0265051707007425$

Hallam, S. (2002). Musical Motivation: Towards a model synthesising the research. Music Education Research, 4(2), 1225-244. doi: 10.1080/1461380022000011939

Kemmis, S. (1989). Investigación en la acción. Enciclopedia Internacional de La Educación, 6, 3330-3337.

Kemmis, S. (2010). What is to be done? The place of action research. Educaction Action Research, 18(4), 417-427. doi: 10.1080/09650792.2010.524745 
Kemmis, S. y McTaggart, R. (1988). Cómo planificar la Investigación-Acción. Barcelona: Laertes.

Knight, P. (2002). A systemic approach to professional development: learning as practice. Teaching and Teacher Education, 18(3), 229-241.

Latorre, A. (2003). La investigación-acción. Conocer y cambiar la práctica educativa. Barceona: Graó.

Lewin, K. (1946). Action research and minority problems. Journal of Social Issues, 2(4), 34-46.

Lucato, M. (2001). El método Kódaly y la formación del profesorado de música. Revista Electrónica de LEEME, (7), 1-7.

McKernan, J. (1999). Investigación-acción y curriculum. Madrid: Morata, S.L.

McPherson, G. E., \& Davidson, J. W. (2006). Playing an instrument. In G. E. Mcpherson (Ed.), The child as musician (pp. 331-351). New York: Oxford University Press.

North, A., \& Hargreaves, D. (2008). The Social and Applied Psychology of Music. New York: Oxford University Press.

O’Neill, S. A., \& Sloboda, J. A. (1997). The Effects of Failure on Children's Ability to Perform a Musical Test. Psychology of Music, 25(1), 18-34.

Odena, O., \& Cabrera, L. (2006). Dramatising the score: an action research investigation of the use of Mozart's Magic Flute as performance guide for his clarinet concert. In 9th International Conference on Music Perception and Cognition (pp. 706-709). University of Bologna, August 22-26.

Pajares, F. (2002). Gender and Perceived self-Efficacy in Self-Regulated Learning. Theory into Practice, 41(2), 116-125.

Porlán, R., \& Martín, J. (1991). El diario del profesor: un recurso para la investigación en el aula. Sevilla: Díada.

Pozo, J.-I., Bautista, A., \& Torrado, J.-A. (2008). El aprendizaje y la enseñanza de la interpretación musical: cambiando las concepciones y las prácticas. Cultura $Y$ Educación, 20(1), 5-15. doi: 10.1174/113564008783781495

Romera, M. J. (2012). La investigación-acción en Didáctica de la Matemáticas: teória y realizaciones. Revista Investigación En La Escuela, (78), 69-80.

Weiner, B. (1985). An Attibutional Theory of Achievement Motivation and Emotion. Psychological Review, 92(4), 548-573.

Weiner, B. (1987). The Role of Emotions in a Theory of Motivation. In F. H. \& J. Kuhl (Ed.), Motivation, Intention, and Volition (pp. 21-30). Berlin: Springer-Verlag.

Wigfield, A., \& Eccles, J. (1994). Children's Competence Beliefs, Achievement Values, and General Self-Esteem. Change Across Elementary and Middle School. Journal of Early Adolescence, 14(2), 107-138.

Zimmerman, B., \& Pons, M. (1988). Construct Validation of a Strategy Model of Student Selfregulated Learning. Journal of Educational Psychology, 80, 284-290. 